\title{
Twin epidemics of new and prevalent hepatitis C infections in Canada: BC Hepatitis Testers Cohort
}

\author{
Naveed Zafar Janjua ${ }^{1,2^{*}}$, Amanda Yuํ, Margot Kuo ${ }^{1}$, Maria Alvarez', Darrel Cook', Jason Wong ${ }^{1}$, \\ Mark W. Tyndall ${ }^{1,2}$ and Mel Krajden ${ }^{1,3}$
}

\begin{abstract}
Background: We characterized the twin epidemics of new and prevalent hepatitis $\mathrm{C}$ virus (HCV) infections in British Columbia, Canada to inform prevention, care and treatment programs.

Methods: The BC Hepatitis Testers Cohort (BC-HTC) includes individuals tested for HCV, HIV or reported as a case of HBV, HCV, HIV or active TB between 1990-2013 linked with data on their medical visits, hospitalizations, cancers, prescription drugs and mortality. Prevalent infection was defined as being anti-HCV positive at first test. Those with a negative test followed by a positive test were considered seroconverters or new infections.

Results: Of 1,132,855 individuals tested for HCV, 64,634 (5.8\%) were positive and an additional 3092 cases tested positive elsewhere for a total of 67,726 . Of 55,781 HCV positive individuals alive at the end of 2013, 7064 were seroconverters while 48,717 had prevalent infection at diagnosis. The HCV positivity rate (11.2\%) was highest in birth cohort 1945-1964 which declined over time. New infections were more likely to be male, 15-34 years of age (born 1965-1984), HIV- or HBV-coinfected, socioeconomically disadvantaged, have problematic drug and alcohol use and a mental health illness. The profile was similar for individuals with prevalent infection, except for lower odds of HBV-coinfection, major mental health diagnoses and birth cohort $>1975$.

Conclusions: The HCV positivity rate is highest in birth cohort 1945-1964 which represents most prevalent infections. New infections occur in younger birth cohorts who are commonly coinfected with HIV and/or HBV, socioeconomically marginalized, and living with mental illness and addictions.
\end{abstract}

Keywords: HCV, Hepatitis B, HIV, Epidemiology, Screening, Cohort study

\section{Background}

Hepatitis C virus (HCV) is a major global public health problem. Worldwide, approximately 184 million people are infected [1], and in Canada, 210,753461,517 (0.66 \%-1.3 \%) people are estimated to be infected with HCV [2]. In British Columbia (BC), the annual rate of newly identified $\mathrm{HCV}$ cases is about $50 \%$ higher than the national average [3]. Untreated $\mathrm{HCV}$ infected individuals have a $\sim 5$-fold increased all-cause and $\sim 20$-fold increased risk of liver-related mortality

\footnotetext{
* Correspondence: naveed.janjua@bccdc.ca

${ }^{1}$ Clinical Prevention Services, British Columbia Centre for Disease Control, Vancouver, BC, Canada

${ }^{2}$ School of Population and Public Health, University of British Columbia,

Vancouver, BC, Canada

Full list of author information is available at the end of the article
}

[4]. While treatment reduces both morbidity and mortality, only about $10 \%$ of infected individuals have been treated in $\mathrm{BC}$ [5]. Newer well-tolerated and highly effective direct acting antiviral agents, with cure rate approaching $95 \%$, are expected to provide new opportunities to prevent progressive liver disease $[6,7]$. However, a large number of people are unaware of their infection $[2,8]$. Characterising individuals who have been tested and who test positive can help identify prevention, testing, care and treatment strategies and programs to reach people most in need.

In most developed countries, the different HCV affected populations can be defined from their risk characteristics that can inform prevention, testing and treatment policies. $\mathrm{HCV}$ affected individuals could be classified into two 
broad groups: prevalent and new infections. Prevalent $\mathrm{HCV}$ infections represent infections acquired in the distant past and these individuals are usually not involved in ongoing risk activities. New infections represent ongoing transmission usually occurring in people with high risk activities such as injection drug use. These two groups differ not only in terms of demographics and risk factors but also have varying levels of onward transmission risk and hence unique needs for engagement in prevention and care. Characterization of these population groups using testing patterns, co-occurrence of other infections (HIV, tuberculosis [TB], sexually transmitted infections [STIs], hepatitis B [HBV]), and social risk factors (addiction and mental illness) could inform the design of prevention, testing and treatment programs tailored to the specific needs of these groups. In this paper, we characterize twin epidemics of $\mathrm{HCV}$ infection by describing characteristics and associated factors of acute and prevalent infections using data from the BC Hepatitis Testers Cohort (BC-HTC). We further characterize the $\mathrm{HCV}$ disease burden by birth cohorts and present positivity by birth cohort to inform birth cohort screening.

\section{Methods}

\section{The cohort and study population}

The BC Hepatitis Testers Cohort(BC-HTC) includes all individuals tested for HCV or HIV at the BC Public Health Laboratory or reported to public health as a confirmed case of HCV, HBV, HIV/AIDS or active TB. The cohort is linked with medical visits, hospitalizations, prescription drugs, cancers and deaths [9-13] (Table 1). Details of the cohort creation, linkage and characteristics of matched and unmatched individuals have been reported previously [14]. The overall linkage rate for $\mathrm{HCV}$ was $>85 \%$ and approached $90 \%$ after 2006.

BC-HTC participants with at least one HCV test (antibody, HCV RNA, or genotype) or HCV case report contributed to the analysis from their first HCV test date (beginning 1992) or report (beginning 1990) through date of death or December 31, 2013, whichever was earliest (Table 1). We excluded data which preceded a participants' birth date or occurred $>7$ days after the death date. Antibody tests in infants $<18$ months in the absence of HCV RNA testing were excluded as passive maternal antibody could not be ruled out.

Table 1 Criteria and Data Sources for the BC Hepatitis Testers Cohort (BC-HTC) and current analysis

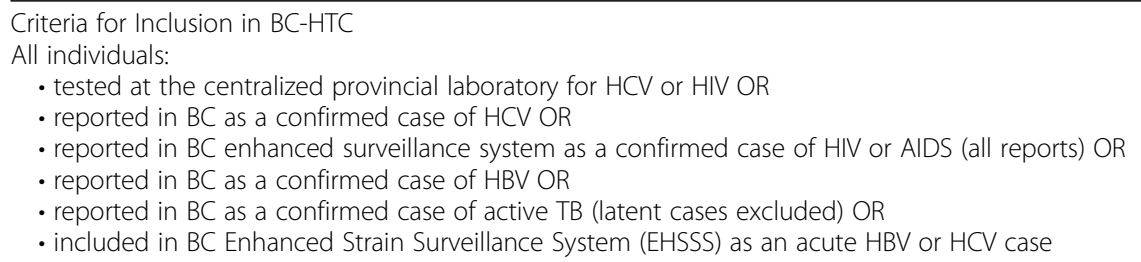

All individuals meeting at least one the above criteria were linked internally across all their tests and case reports. Those with a valid personal health number (PHN) were then sent for deterministic linkage with province-wide Cancer and Ministry of Health $(\mathrm{MoH})$ datasets

Provincial Communicable Disease Data Sources

- BC-PHL HIV laboratory testing datasets (tests: ELISA, Western blot, NAAT, p24, culture)

- BC-PHL HCV laboratory tests datasets (tests: antibody, HCV RNA, genotyping)

- HIV/AIDS Information System (HAISYS) (public health HIV/AIDS case reports)

- Integrated Public Health information System (iPHIS) (public health case reports of HCV, HBV, and TB)

- Enhanced Strain Surveillance System (EHSSS) (risk factor data on a subset of acute HCV and acute HBV cases)

Data Date Ranges: HIV lab data: 1988-2013 HCV lab data: 1992-2013 HAISYS: $1980-2013$ iPHIS: $1990-2013$ EHSSS: 2000-2013

Cancer and $\mathrm{MoH}$ Administrative Data Sources

- BC Cancer Registry (BCCR) (primary tumour registry, excludes metastatic cancers)

- Discharge Abstracts Dataset (DAD) (hospitalization records)

- Medical Services Plan (MSP) (physician diagnostic and billing data)

- PharmaCare/PharmaNet (Pharma) (prescription drug dispensations)

- BC Vital Statistics (VS) (deaths registry)
Data Date Ranges: BCCR: 1970-2012 DAD: $1985-2013 Q 1$ MSP: $1990-2012$

Pharma: 1985-2012

VS: $1985-2013$

The final BC-HTC comprises all individuals successfully linked on PHN to the MoH Client Roster (a registry of all BC residents enrolled in the publicly-funded universal healthcare system)

\footnotetext{
Eligibility for current analysis

- all subjects included in final, linked BC-HTC with at least one valid HCV test (antibody, HCV RNA, or genotype) (1992-2013)

or HCV case report (1990-2013)

- subjects were censored for analysis date of death or December 31,2013, whichever came first

- excluded all data preceding subject's recorded date of birth

- excluded all data occurring more than 7 days after subject's recorded date of death

- excluded HCV antibody tests in infants less than 18 months in the absence of HCV RNA confirmatory testing
} 


\section{Definitions}

A HCV case was defined as an individual testing positive for either $\mathrm{HCV}$ antibody or RNA or genotype or was reported as a $\mathrm{HCV}$ case through the public health reporting system [15]. We considered participants who were $\mathrm{HCV}$ antibody positive at their first test on record as having prevalent infection as, without a prior negative test, a seroconversion interval cannot be established and, based on natural history, most will be chronically infected. Those cases not found in laboratory data but reported by public health as confirmed $\mathrm{HCV}$ were considered prevalent infections. Individuals with a negative followed by a positive test were considered seroconverters or new infections. Individuals who seroconverted within 24 months of the last negative test were termed 24-month seroconverters, while those who seroconverted after 24 months are >24-months seroconverters. For surveillance purposes in BC, acute $\mathrm{HCV}$ infection is defined as seroconversion within the previous 12 months [15]. Since the characteristics of 12- and 24-month seroconverters were similar as presented in Additional file 1: Table S2, we report 0-24month seroconverters as acute HCV cases. Co-infection was defined as being infected with HIV, HBV or active TB together with HCV.

Socioeconomic status was assessed using the Québec Index of Material and Social Deprivation, a widely used index based on Canadian Census data [16]. Data from each census, conducted every 5 years, was applied to two years before and two years after the census year. For the 2011 census, the index is based on the data product CensusPlus to address non-response related bias in the 2011 National Household Survey [17].

Assessment of illicit drug use, mental illnesses, and problematic alcohol use was based on having diagnostic codes in administrative health datasets evaluated across all data prior to and within 3 years of the first positive test or last negative test (Additional file 1: Table S1).

\section{Statistical analysis}

We compared the characteristics of $\mathrm{HCV}$ positive cases (24-month seroconverters, >24-month seroconverters and prevalent cases) and negative groups using the chisquare test for categorical factors or the WilcoxonMann-Whitney test for continuous variables. We calculated HCV positivity across birth cohorts and compared their characteristics. Positivity rate is defined as number of hepatitis $\mathrm{C}$ diagnoses divided by the total number of people tested for hepatitis C. We performed multinomial logistic regression to assess the factors associated with new and prevalent infections at diagnosis compared to HCV negatives.

\section{Results}

Participant Profile, testing and infection prevalence

The cohort included 1,132,855 subjects tested for hepatitis C from 1992-2013. Of these, 64,634 (5.7\%) were $\mathrm{HCV}$ positive. An additional 3092 cases tested elsewhere and reported to the public health surveillance system from 1990-2013 resulted in a total of 67,726 HCV cases. Of $\mathrm{HCV}$ positive individuals, 11,945 (17.6\%) have died and 55,781 were alive at the end of 2013. Among 1,068,221 HCV negative individuals, 79,840 (7.5 \%) have died and 988,381 were alive at the end of 2013. The following results are from individuals alive as of December 31, 2013 unless specified otherwise.

\section{Characteristics of HCV positive and negative individuals}

For $\mathrm{HCV}$ positive individuals, the median age at their first test was 40 years and 35 years for $\mathrm{HCV}$ negative individuals. A comparatively small proportion (12.2\%) of $\mathrm{HCV}$ positive individuals were born after 1975 compared to those $\mathrm{HCV}$ negative $(37.6 \% ; p<0.001)$. A higher proportion of $\mathrm{HCV}$ positives compared to negatives were male (64.4 \% vs. $44.1 \% ; p<0.001)$. Infection with HIV (5.7 \% vs. $0.5 \% ; p<0.001)$, HBV (3.7 \% vs. $2.0 \% ; p<0.001)$ and active TB $(0.5 \%$ vs $0.2 \% ; p<0.001)$ were significantly higher in $\mathrm{HCV}$ positives than negatives. There was a graded increase in percent $\mathrm{HCV}$ positivite with increases in material (most privileged: $3.2 \%$ vs. most deprived: $8.0 \%$ ) and social deprivation (most privileged: $3.1 \%$ vs. most deprived: $7.7 \%$ ). There were fewer privileged (material: 13.4 \% vs. $22.0 \%$ /social: $11.0 \%$ vs $18.5 \%$ ) and more deprived (material: $29.6 \%$ vs. $18.8 \%$; $p<0.001 /$ social: $37.7 \%$ vs. $24.8 \% ; p<0.001)$ individuals among HCV positives compared to HCV negatives. The proportions with illicit drug use (35.2 \% vs. $7.0 \%)$, problematic alcohol use (21.7 \% vs. $6.2 \%)$ and mental health conditions (16.0 \% vs. $12.0 \%)$ at diagnosis were also higher among $\mathrm{HCV}$ positives compared to HCV negatives (Table 2).

\section{Seroconversion and characteristics of new and prevalent HCV cases}

Of 370,741 individuals who tested for anti-HCV multiple times since 1992, 7726 (2.1 \%) seroconverted. Among the 346,428 currently alive repeat testers, $6922(2.0 \%)$ seroconverted. Among those testing only once $(n=$ $762,114), 56,908$ (7.5 \%) tested positive (prevalent HCV infections). Of these, 695,137 were alive at the end of 2013 and 46,262 (6.7\%) were positive.

Of the 6922 currently alive seroconverters, 1989 (28.7\%) seroconverted within 12 months, 1497 (21.6 \%) in 12-24 months and 3436 (49.6\%) >24-months after their last negative test (Additional file 1: Table S2).

24-month seroconverters and reported acute cases were younger than both $>24$-month seroconverters and individuals with prevalent infection (median ages: 30, 35, 
Table 2 Characteristics of HCV positive and negative individuals, BC-HTC, Canada, 1990-2013

\begin{tabular}{|c|c|c|c|c|c|}
\hline & \multicolumn{4}{|c|}{$\mathrm{HCV}+$ ve group } & \multirow{2}{*}{$\begin{array}{l}\text { HCV -ve group } \\
\text { All Negatives }\end{array}$} \\
\hline & M24 Sero ${ }^{a}$ & $>$ M24 Sero ${ }^{b}$ & Prevalent HCV & All Positives & \\
\hline & $N=3628$ & $N=3436$ & $N=48717$ & $N=55781$ & $N=988381$ \\
\hline & N (\%) & N (\%) & N (\%) & N (\%) & N (\%) \\
\hline \multicolumn{6}{|l|}{ Age at baseline ${ }^{c}$} \\
\hline$<15$ & $17(0.5)$ & $1(0.0)$ & $420(0.9)$ & $438(0.8)$ & $20289(2.1)$ \\
\hline $15-24$ & $962(26.5)$ & 410 (11.9) & $2410(5.0)$ & $3782(6.8)$ & $145326(14.7)$ \\
\hline $25-34$ & $1477(40.7)$ & $1234(35.9)$ & $9907(20.3)$ & $12618(22.6)$ & $259347(26.2)$ \\
\hline $35-44$ & $827(22.8)$ & $1106(32.2)$ & $16534(33.9)$ & $18467(33.1)$ & 220626 (22.3) \\
\hline $45-54$ & $275(7.6)$ & $522(15.2)$ & $13068(26.8)$ & 13865 (24.9) & $163134(16.5)$ \\
\hline$\geq 55$ & $70(1.9)$ & $163(4.7)$ & $6378(13.1)$ & $6611(11.9)$ & 179659 (18.2) \\
\hline Median [IQR] & 30 [24-37] & 35 [28-43] & 42 [34-49] & 41 [33-48] & 37 [28-50] \\
\hline \multicolumn{6}{|l|}{ Age at $1 \mathrm{st}$ test } \\
\hline$<15$ & $100(2.8)$ & $84(2.4)$ & $421(0.9)$ & $605(1.1)$ & $25860(2.6)$ \\
\hline $15-24$ & 1485 (40.9) & 1128 (32.8) & $2422(5.0)$ & $5035(9.0)$ & 189747 (19.2) \\
\hline $25-34$ & 1225 (33.8) & $1278(37.2)$ & $9927(20.4)$ & $12430(22.3)$ & 261056 (26.4) \\
\hline $35-44$ & $605(16.7)$ & $712(20.7)$ & $16590(34.1)$ & 17907 (32.1) & 208398 (21.1) \\
\hline $45-54$ & $169(4.7)$ & $195(5.7)$ & $13063(26.8)$ & $13427(24.1)$ & $147688(14.9)$ \\
\hline$\geq 55$ & $44(1.2)$ & $39(1.1)$ & $6294(12.9)$ & $6377(11.4)$ & 155632 (15.8) \\
\hline Median [IQR] & 26 [20-34] & 28 [22-35] & 42 [34-49] & 40 [32-48] & 35 [26-48] \\
\hline \multicolumn{6}{|l|}{ Birth year } \\
\hline$<1945$ & $31(0.9)$ & $35(1.0)$ & $3102(6.4)$ & $3168(5.7)$ & $95848(9.7)$ \\
\hline 1945-1964 & $776(21.4)$ & $1037(30.2)$ & $32133(66.0)$ & $33946(60.9)$ & 310845 (31.5) \\
\hline 1965-1974 & $1176(32.4)$ & $1214(35.3)$ & 9493 (19.5) & $11883(21.3)$ & 209956 (21.2) \\
\hline$\geq 1975$ & 1645 (45.3) & 1150 (33.5) & $3989(8.2)$ & $6784(12.2)$ & 371732 (37.6) \\
\hline \multicolumn{6}{|l|}{ Sex } \\
\hline Female & $1728(47.6)$ & $1380(40.2)$ & $16741(34.4)$ & 19849 (35.6) & $551952(55.8)$ \\
\hline Male & $1900(52.4)$ & $2056(59.8)$ & $31970(65.6)$ & $35926(64.4)$ & $436287(44.1)$ \\
\hline Unknown & $0(0.0)$ & $0(0.0)$ & $6(0.0)$ & $6(0.0)$ & $142(0.0)$ \\
\hline \multicolumn{6}{|l|}{ Year of diagnosis } \\
\hline 1990-1994 & $85(2.3)$ & $4(0.1)$ & $3978(8.2)$ & $4067(7.3)$ & $3913(0.4)$ \\
\hline 1995-1999 & $726(20.0)$ & $353(10.3)$ & $17936(36.8)$ & $19015(34.1)$ & $104863(10.6)$ \\
\hline $2000-2004$ & 1177 (32.4) & $939(27.3)$ & $11733(24.1)$ & $13849(24.8)$ & 172888 (17.5) \\
\hline $2005-2009$ & $994(27.4)$ & $1280(37.3)$ & $9130(18.7)$ & $11404(20.4)$ & 272886 (27.6) \\
\hline 2010-2013 & $646(17.8)$ & $860(25.0)$ & $5940(12.2)$ & $7446(13.4)$ & 433831 (43.9) \\
\hline \multicolumn{6}{|c|}{ HIV/AIDS at baseline } \\
\hline Unknown & $3450(95.1)$ & 3207 (93.3) & $47568(97.6)$ & $54225(97.2)$ & 984182 (99.6) \\
\hline Yes & $178(4.9)$ & $229(6.7)$ & $1149(2.4)$ & $1556(2.8)$ & $4199(0.4)$ \\
\hline \multicolumn{6}{|l|}{ HIV/AIDS (ever) } \\
\hline Unknown & $3264(90.0)$ & 3104 (90.3) & $46257(95.0)$ & $52625(94.3)$ & 983694 (99.5) \\
\hline Yes & $364(10.0)$ & $332(9.7)$ & $2460(5.0)$ & $3156(5.7)$ & $4687(0.5)$ \\
\hline \multicolumn{6}{|l|}{ HBV at baseline } \\
\hline No/Unknown & $3528(97.2)$ & 3349 (97.5) & $48197(98.9)$ & $55074(98.7)$ & 974223 (98.6) \\
\hline Yes & $100(2.8)$ & $87(2.5)$ & $520(1.1)$ & 707 (1.3) & $14158(1.4)$ \\
\hline
\end{tabular}


Table 2 Characteristics of HCV positive and negative individuals, BC-HTC, Canada, 1990-2013 (Continued)

\begin{tabular}{|c|c|c|c|c|c|}
\hline \multicolumn{6}{|l|}{ HBV (ever) } \\
\hline No/Unknown & $3453(95.2)$ & $3326(96.8)$ & $46927(96.3)$ & $53706(96.3)$ & $968459(98.0)$ \\
\hline Yes & $175(4.8)$ & $110(3.2)$ & $1790(3.7)$ & $2075(3.7)$ & $19922(2.0)$ \\
\hline \multicolumn{6}{|l|}{ Active TB at baseline } \\
\hline No/Unknown & $3621(99.8)$ & $3428(99.8)$ & $48634(99.8)$ & $55683(99.8)$ & $986909(99.9)$ \\
\hline Yes & $7(0.2)$ & $8(0.2)$ & $83(0.2)$ & $98(0.2)$ & $1472(0.1)$ \\
\hline \multicolumn{6}{|l|}{ Active TB (ever) } \\
\hline No/Unknown & $3611(99.5)$ & 3415 (99.4) & $48466(99.5)$ & $55492(99.5)$ & $986473(99.8)$ \\
\hline Yes & $17(0.5)$ & $21(0.6)$ & $251(0.5)$ & $289(0.5)$ & $1908(0.2)$ \\
\hline \multicolumn{6}{|c|}{ Material deprivation quintile at baseline } \\
\hline Unknown & $96(2.7)$ & $79(2.3)$ & $2316(4.8)$ & $2491(4.5)$ & $14773(1.5)$ \\
\hline Q1 (most privileged) & $452(12.5)$ & $444(12.9)$ & $6247(12.8)$ & $7143(12.8)$ & $214007(21.7)$ \\
\hline Q2 & $526(14.5)$ & $463(13.5)$ & $7500(15.4)$ & $8489(15.2)$ & $187587(19)$ \\
\hline Q3 & $531(14.6)$ & $585(17.0)$ & $8522(17.5)$ & $9638(17.3)$ & $192265(19.5)$ \\
\hline Q4 & $876(24.2)$ & $774(22.5)$ & $10580(21.7)$ & $12230(21.9)$ & $197116(19.9)$ \\
\hline Q5 (most deprived) & $1147(31.6)$ & $1091(31.8)$ & $13552(27.8)$ & $15790(28.3)$ & $182633(18.5)$ \\
\hline \multicolumn{6}{|c|}{ Social deprivation quintile at baseline } \\
\hline Unknown & $96(2.7)$ & $79(2.3)$ & $2316(4.8)$ & $2491(4.5)$ & $14773(1.5)$ \\
\hline Q1 (most privileged) & $271(7.5)$ & $283(8.2)$ & $5302(10.9)$ & $5856(10.5)$ & $180214(18.2)$ \\
\hline Q2 & $349(9.6)$ & $337(9.8)$ & $6194(12.7)$ & $6880(12.3)$ & $175098(17.7)$ \\
\hline Q3 & $540(14.9)$ & $463(13.5)$ & 8094 (16.6) & $9097(16.3)$ & $175715(17.8)$ \\
\hline Q4 & $703(19.4)$ & $720(21.0)$ & $9972(20.5)$ & $11395(20.4)$ & $201551(20.4)$ \\
\hline Q5 (most deprived) & $1669(46.0)$ & $1554(45.2)$ & $16839(34.6)$ & $20062(36)$ & $241030(24.4)$ \\
\hline \multicolumn{6}{|l|}{ Mental health at baseline ${ }^{d}$} \\
\hline No/Unknown & $2199(64.5)$ & $2096(65.0)$ & $40934(86.7)$ & $45229(84.0)$ & $729398(88.0)$ \\
\hline Yes & $1209(35.5)$ & $1130(35.0)$ & $6297(13.3)$ & $8636(16.0)$ & $99054(12.0)$ \\
\hline \multicolumn{6}{|c|}{ Mental health 3yrs pre-baseline ${ }^{d}$} \\
\hline No/Unknown & $2590(76.0)$ & $2653(82.2)$ & $43518(92.1)$ & $48761(90.5)$ & $781042(94.3)$ \\
\hline Yes & $818(24.0)$ & $573(17.8)$ & $3713(7.9)$ & $5104(9.5)$ & $47410(5.7)$ \\
\hline \multicolumn{6}{|c|}{ Illicit drug use at baseline ${ }^{d}$} \\
\hline No/Unknown & $910(26.7)$ & $942(29.2)$ & $33036(69.9)$ & $34888(64.8)$ & $770499(93.0)$ \\
\hline Yes & $2498(73.3)$ & $2284(70.8)$ & $14195(30.1)$ & $18977(35.2)$ & $57953(7.0)$ \\
\hline \multicolumn{6}{|c|}{ Illicit drug use 3yrs pre-baseline ${ }^{d}$} \\
\hline No/Unknown & $1084(31.8)$ & $1433(44.4)$ & $36193(76.6)$ & $38710(71.9)$ & $795325(96.0)$ \\
\hline Yes & $2324(68.2)$ & $1793(55.6)$ & $11038(23.4)$ & $15155(28.1)$ & $33127(4.0)$ \\
\hline \multicolumn{6}{|c|}{ Problematic alcohol use at baseline ${ }^{d}$} \\
\hline No/Unknown & $2083(61.1)$ & $1896(58.8)$ & $38178(80.8)$ & $42157(78.3)$ & $777271(93.8)$ \\
\hline Yes & $1325(38.9)$ & $1330(41.2)$ & $9053(19.2)$ & $11708(21.7)$ & $51181(6.2)$ \\
\hline \multicolumn{6}{|c|}{ Problematic alcohol use 3yrs pre-baseline ${ }^{d}$} \\
\hline No/Unknown & $2521(74.0)$ & $2555(79.2)$ & $41709(88.3)$ & $46785(86.9)$ & $802492(96.9)$ \\
\hline Yes & $887(26.0)$ & $671(20.8)$ & $5522(11.7)$ & $7080(13.1)$ & $25960(3.1)$ \\
\hline
\end{tabular}

a24-month Sero: Individuals who seroconverted within 24 months of the last negative test

${ }^{b}$ Individuals who seroconverted after 24 months of the last negative test

'Baseline is defined as date of diagnosis (i.e. first HCV positive test or case report) for HCV positive individuals, and date of last negative test result for HCV

negative individuals

${ }^{\mathrm{d}}$ Mental health, drug misuse, alcohol misuse data was available up to 2012 
42 years; $p<0.001)$. Most of the individuals with prevalent infection (66.0\%) were born between 1945-64 with another $19.5 \%$ between 1965-74. Of 24-month seroconverters, $32.4 \%$ were born in 1965-74, and $45.3 \%$ after 1975. A higher percentage of prevalent cases and $>24$ month seroconverters were male compared to 24-month seroconverters (65.6\%, $59.8 \%$ vs $52.4 \%$ ) (Table 2 ).

HIV and HBV co-infections were higher among 24month seroconverters compared to prevalent $\mathrm{HCV}$ cases (HIV: $10.0 \%$ vs. $5.1 \%$; HBV: $4.8 \%$ vs. $3.7 \%$ ) while the proportion with active TB was the same (0.5\% vs. $0.5 \%)$ (Fig. 1a). The proportions with illicit drug use (73.3\% vs. $30.1 \%)$, problem alcohol use (38.9\% vs. $19.2 \%)$ and mental health conditions (35.5\% vs. $13.3 \%$ ) were also higher among 24-month seroconverters than individuals with prevalent HCV infection (Table 2, Fig. 1b).

Characteristics of participants diagnosed in recent years (2010-2013) are presented in Additional file 1: Table S3. Overall characteristics are similar with differences in age at diagnosis, birth cohort, HIV, HBV infection, and mental illness mainly in 24 month seroconverters.

\section{HCV infection by birth cohort}

$\mathrm{HCV}$ positivity rates showed a strong birth cohort effect (Fig. 2a, b Additional file 1: Figure S1). Birth cohorts 1950-1954 and 1955-59 had the highest percentage positive overall followed by 1960-64 though positivity declined over time and was lowest in 2013. Birth cohorts 1945-49

a

80

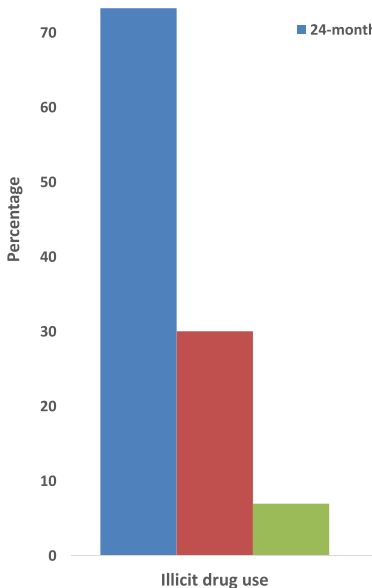

- Prevalent HCV = HCV negative

b

12

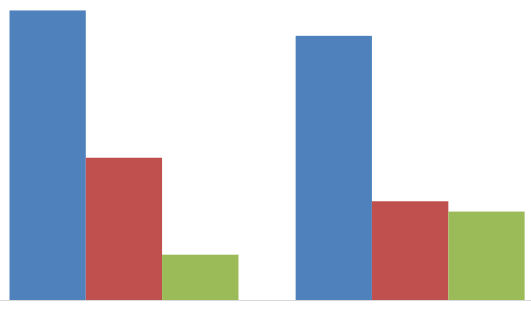

Problem alcohol use

Mental illness
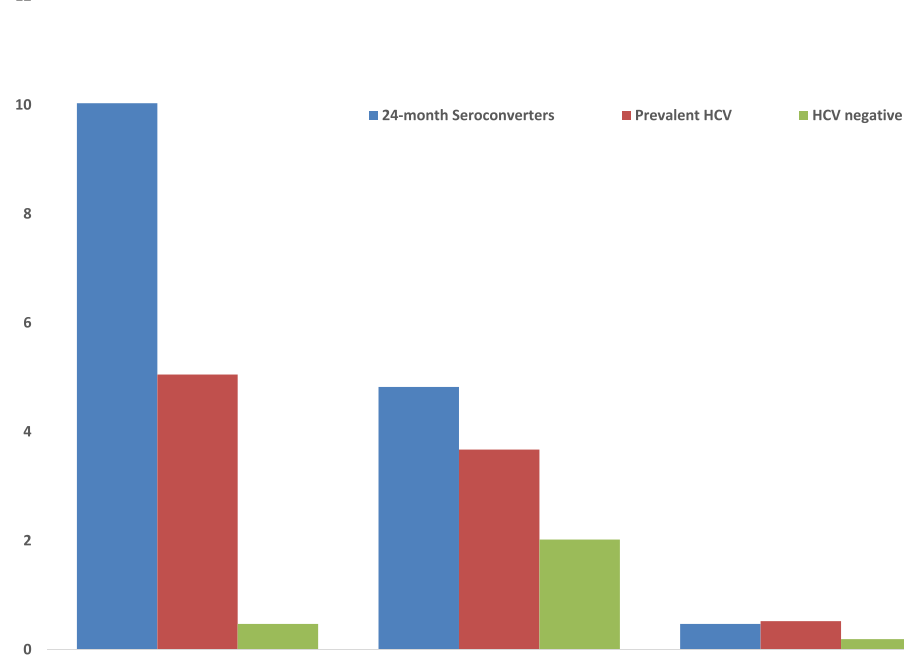

HIV

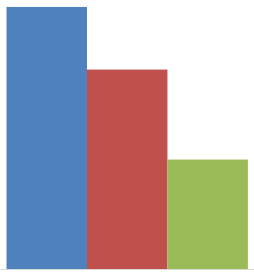

HBV

Active TB

Fig. 1 a Distribution of illicit drug use, alcohol use, and mental illness by HCV infection status in BC-HTC, Canada 1990-2013. b Distribution of co-infections by HCV infection status in BC-HTC, Canada 1990-2013 
a —All HCV positive -Prevalent HCV —Seroconverters

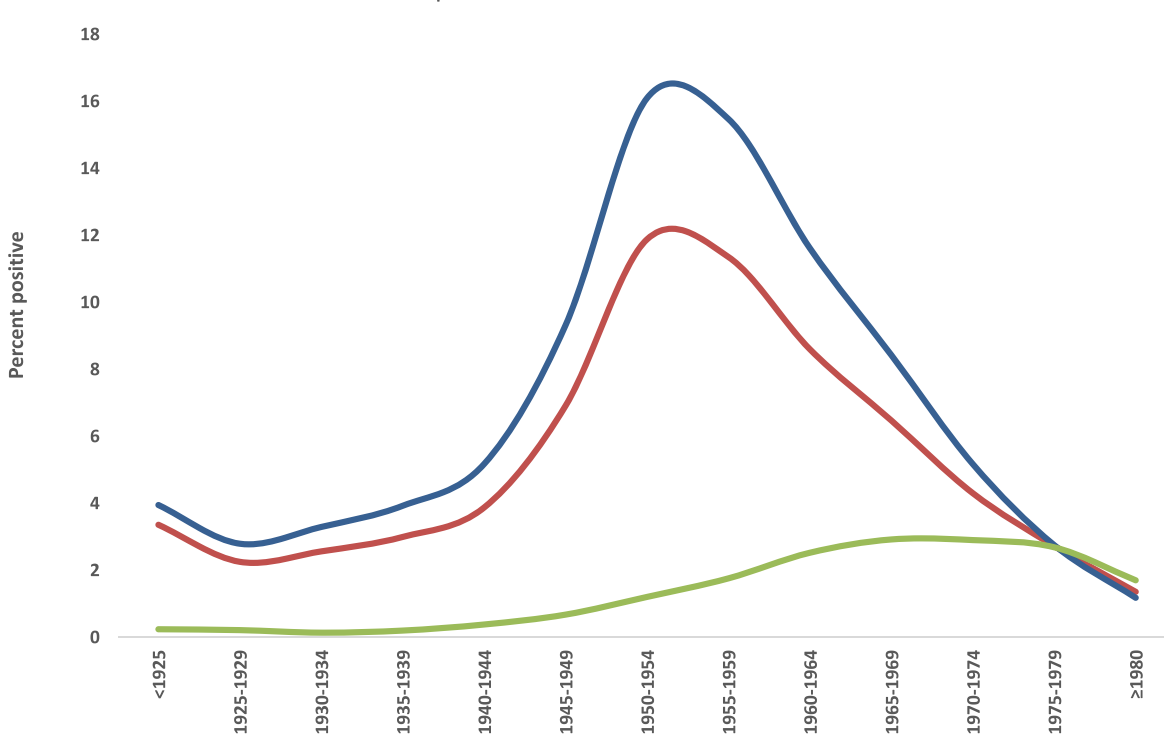

b

Birth Cohort

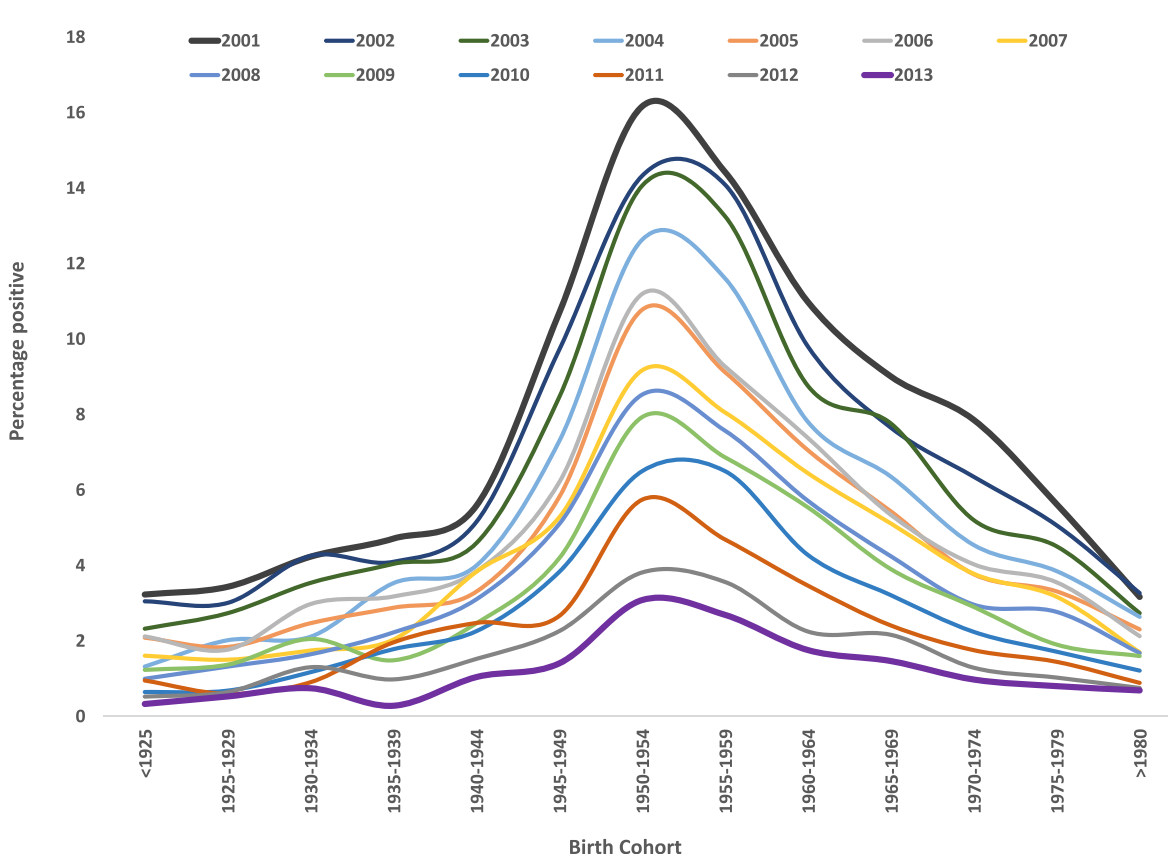

Fig. 2 a Hepatitis C positivity by birth cohort in BC-HTC, British Columbia, Canada 1990-2013. HCV positives include both seroconverters and chronic HCV. b Hepatitis C positivity by birth cohort and year in BC-HTC, British Columbia, Canada, 2001-2013

and 1965-69 had similar percentage positive though it was slightly higher for 1945-49. In 2013, there were 1,262,121 people born between 1945 and 1964 living in BC; 344,791 (27.3 \%) have been tested and 33,946 (9.8 \%) were HCV positive, of whom 7352 (17.8 \%) have already died. Assuming constant in and outflow, about $70 \%$ of 1945-64 and 1950-70 birth cohorts have not been tested for HCV.
In birth cohort 1945-64, $67.8 \%$ were male compared to $63.9 \%$ and $51.9 \%$ of those born $1965-74$ and after 1975 , respectively. A small proportion (2.3\%) of 24month seroconverters was identified among HCV positives born 1945-64 compared to $9.9 \%$ and $24.3 \%$ among 1965-1974 and $\geq 1975$ birth cohorts, respectively. In contrast, $97.9 \%, 94.7 \%, 79.9 \%$ and $58.8 \% \mathrm{HCV}$ positive tests 
were detected on the first test (prevalent infections) among those born $<1945$, 1945-64, 1965-1974, and $\geq 1975$, respectively (Table 3 ).

The proportion of $\mathrm{HCV} / \mathrm{HIV}$ co-infected individuals increased with increasing birth year $(<1945: 1.0 \%$, 194564: $4.7 \%, 1965-1974: 9.0 \%$ and >1975: $6.6 \%)$. In contrast, among HCV negative individuals, the HIV infection rates by birth cohort were higher among those born between 1945-64 and 1965-74. HBV co-infection was $2.9 \%, 3.8 \%, 4.7 \%$ and $1.9 \%$ among those born $<1945,1945-64,1965-1974$, and $\geq 1975$, respectively. Illicit drug use (36.7\% vs $10.4 \%)$, problematic alcohol use (22.3 $\%$ vs $11.7 \%)$ and mental health conditions (16.4\% vs 10.2 $\%)$ were higher among those born after 1945 than those born before 1945 (Table 3).

Factors associated with new and prevalent $\mathrm{HCV}$ infection In the multivariable multinomial logistic regression model (Table 4), new HCV infections were associated with being male, born after 1945, HIV co-infection, HBV co-infection, material deprivation, illicit drug use, problematic alcohol use and mental health diagnoses. The same factors were associated with prevalent HCV infections except for lower odds for HBV co-infection, mental health conditions and birth cohort $\geq 1975$. The odds of prevalent infection were highest in the 1945-54 birth cohort (OR: 3.8, 95 \% CI: 3.7-3.9) followed by 1955-64 (OR: 3.2, 95 \% CI: 3.1-3.3), while the odds of being a new infection were highest for the 1975-84 (OR: 11.8, 95 \% CI: 9.9-14.1) birth cohort followed by those born 1965-74 (OR: 9.9, 95 \% CI: 8.3-11.8). Furthermore, odds ratios for illicit drug use (ORs: 21.0, 95 \% CI: 19.8-22.4 vs. 5.0, $95 \%$ CI: 4.9-5.2) and HIV co-infection (ORs: 7.2, 95 \% CI: 7.18.9 vs. $4.8,95 \%$ CI: 4.5-5.2) were much higher for new infections than for prevalent $\mathrm{HCV}$ infections. In a model with age at diagnosis instead of birth cohort (Additional file 1: Table S5), odds of prevalent infection were highest among ages 45-54 years (OR: 4.0; 95 \% CI: 3.9-4.2) followed by $35-44$ years (OR: 3.2; 95 \% CI: 3.0-3.3) while odds for new infection were highest among ages 15-24 years (OR: 11.4, 95 \% CI: 9.0-14.4) and 25-34 years (OR: 11.1, 95 \% CI: 8.8-14.0) and declined thereafter. Similar results were observed using indicators for recent risk activities (Additional file 1: Table S6).

\section{Discussion}

We used a large cohort of more than one million people tested for $\mathrm{HCV}$ in $\mathrm{BC}$, and while accounting for mortality, we found that a large number of people are currently living with HCV. The highest positivity rate was among people born in 1945-64, which declined over time and was lowest in 2013. New infections were detected mainly among younger age groups. The new $\mathrm{HCV}$ infection (seroconversion) rate was highest among males, those with HIV or HBV co-infection, mental health conditions, problematic alcohol or illicit drug use, and socioeconomically disadvantaged persons. Prevalent HCV infection was associated with being male, born 1945-64, HIV co-infected, problem alcohol and illicit drug use, and socioeconomic deprivation. These findings of twin epidemics highlight important opportunities for prevention, testing and treatment of $\mathrm{HCV}$.

In this analysis, we identified two groups of HCV infected individuals: new infections (seroconverters) and those with prevalent $\mathrm{HCV}$ infection. Most of those with prevalent HCV were born 1945-1964, while most newly infected individuals were younger. However, the seroconverters are not a homogeneous group: 24-month seroconverters differed from $>24$ months seroconverters in terms of age, sex, testing patterns, and HBV co-infection rate. Surveillance data on acute $\mathrm{HCV}$ infections indicate that $70 \%$ of seroconverters reported injection drug use in the past 12 months [18]. In a BC-HTC subset for whom self-reported risk factor data was available, $85 \%$ of those who self-reported injection drug use had a medical visit for illicit drug use. Thus, 24-month seroconverters most likely acquired $\mathrm{HCV}$ through their injection drug use networks.

Most of the HCV cases, especially prevalent infections, were identified among those born 1945-64 and the positivity rate was much higher in this group, especially for those born 1950-1960. Similarly high 1945-64 birth cohort positivity rates have been reported in various studies from the United States [19-22]. Using reported HCV cases in Canada, Trubnikov et al. found the highest HCV prevalence among the 1950-54 and 1955-59 birth cohorts, followed by 1960-64 and 1966-69, while prevalence among those born 1945-49 was lower than for those born 1965-69 [2]. In contrast, in our study, the positivity rate for birth cohort 1945-49 was higher than for birth cohort 1965-69 (Fig. 2). Our findings are also consistent with a study which assessed HCV related hospital admissions where the 1950-54 and 1955-59 birth cohorts had the highest rates [23]. Although the positivity rate is still highest among the 1945-64 birth cohort, the rate in this cohort has been declining over successive years suggesting a decreasing pool of undiagnosed prevalent HCV infections. Canada has had risk based testing guidelines since 1994 [24]. The declining positivity rate in this birth cohort suggests that testing driven by risk based guidelines have been able to identify most people with current or past risk activities and diagnose most of the HCV infections in this cohort. However, risk based testing may not identify individuals who are unable to recall or are unwilling to disclose remote risk behaviors. The US Preventive Services Task Force has recommended one time testing of individuals born 1945-65 [22]. Further 
Table 3 Characteristics of currently alive HCV testers by HCV status and birth cohort, Canada, BC-HTC, 1990-2013

\begin{tabular}{|c|c|c|c|c|c|c|c|c|}
\hline & \multicolumn{4}{|l|}{ HCV Positive } & \multicolumn{4}{|l|}{ HCV Negative } \\
\hline & $<1945$ & 1945-1964 & 1965-1974 & $\geq 1975$ & $<1945$ & 1945-1964 & 1965-1974 & $\geq 1975$ \\
\hline & $N=3168$ & $N=33946$ & $N=11883$ & $N=6784$ & $N=95848$ & $N=310845$ & $N=209956$ & $N=371732$ \\
\hline & N (\%) & N (\%) & $N(\%)$ & N (\%) & N (\%) & $N(\%)$ & N (\%) & N (\%) \\
\hline \multicolumn{9}{|l|}{ Age at baseline ${ }^{a}$} \\
\hline$<15$ & 0 & 0 & 0 & $438(6.5)$ & 0 & 0 & 0 & $20289(5.5)$ \\
\hline $15-24$ & 0 & 0 & $857(7.2)$ & $2925(43.1)$ & 0 & 0 & 3591 (1.7) & $141735(38.1)$ \\
\hline $25-34$ & 0 & $2764(8.1)$ & $6688(56.3)$ & $3166(46.7)$ & 0 & $6369(2.1)$ & $66420(31.6)$ & $186558(50.2)$ \\
\hline $35-44$ & 0 & $14198(41.8)$ & 4014 (33.8) & 255 (3.8) & 0 & $73286(23.6)$ & $124190(59.2)$ & $23150(6.2)$ \\
\hline $45-54$ & $425(13.4)$ & $13116(38.6)$ & $324(2.7)$ & 0 & 3166 (3.3) & $144213(46.4)$ & $15755(7.5)$ & 0 \\
\hline$\geq 55$ & $2743(86.6)$ & $3868(11.4)$ & 0 & 0 & $92682(96.7)$ & $86977(28.0)$ & 0 & 0 \\
\hline Median [IQR] & $63[58-70]$ & 45 [39-50] & $32[28-37]$ & 25 [21-29] & 69 [63-75] & 50 [44-55] & 37 [33-41] & 26 [21-30] \\
\hline \multicolumn{9}{|l|}{ Age at $1 \mathrm{st}$ test } \\
\hline$<15$ & 0 & 0 & 0 & 605 (8.9) & 0 & 0 & 0 & $25860(7)$ \\
\hline $15-24$ & 0 & 0 & $1205(10.1)$ & $3830(56.5)$ & 0 & 0 & 7867 (3.8) & $181880(48.9)$ \\
\hline $25-34$ & 0 & $3074(9.1)$ & $7174(60.4)$ & $2182(32.2)$ & 0 & $12382(4.0)$ & $96703(46.1)$ & $151971(40.9)$ \\
\hline $35-44$ & 0 & $14485(42.7)$ & 3255 (27.4) & $167(2.5)$ & 0 & 99462 (32.0) & 96915 (46.2) & $12021(3.2)$ \\
\hline $45-54$ & 436 (13.8) & $12742(37.5)$ & $249(2.1)$ & 0 & $5217(5.4)$ & $134000(43.1)$ & $8471(4.0)$ & 0 \\
\hline$\geq 55$ & $2732(86.2)$ & $3645(10.7)$ & 0 & 0 & $90631(94.6)$ & $65001(20.9)$ & 0 & 0 \\
\hline Median [IQR] & $63[58-69]$ & 44 [39-50] & 31 [27-36] & 22 [18-27] & 67 [61-73] & $48[42-53]$ & 35 [30-39] & 24 [20-28] \\
\hline \multicolumn{9}{|l|}{ Sex } \\
\hline Female & $1354(42.7)$ & $10946(32.3)$ & $4288(36.1)$ & 3261 (48.1) & $51063(53.3)$ & $164778(53.0)$ & 116339 (55.4) & $219772(59.1)$ \\
\hline Male & $1809(57.1)$ & $23000(67.8)$ & 7594 (63.9) & $3523(51.9)$ & $44657(46.6)$ & $146061(47.0)$ & $93615(44.6)$ & $151954(40.9)$ \\
\hline Unknown & $5(0.2)$ & 0 & 0 & 0 & $128(0.1)$ & $6(0.0)$ & $2(0.0)$ & $6(0.0)$ \\
\hline \multicolumn{9}{|l|}{ Year of diagnosis } \\
\hline 1990-1994 & $340(10.7)$ & $2938(8.7)$ & $690(5.8)$ & $99(1.5)$ & $822(0.9)$ & $1858(0.6)$ & $889(0.4)$ & $344(0.1)$ \\
\hline 1995-1999 & $1186(37.4)$ & $12980(38.2)$ & 3992 (33.6) & $857(12.6)$ & $21815(22.8)$ & $48746(15.7)$ & $21003(10.0)$ & 13299 (3.6) \\
\hline $2000-2004$ & $703(22.2)$ & $8333(24.6)$ & $3163(26.6)$ & $1650(24.3)$ & $19159(20.0)$ & 68035 (21.9) & $43673(20.8)$ & $42021(11.3)$ \\
\hline 2005-2009 & $555(17.5)$ & $6004(17.7)$ & $2588(21.8)$ & 2257 (33.3) & 22709 (23.7) & $81513(26.2)$ & $63756(30.4)$ & $104908(28.2)$ \\
\hline 2010-2013 & $384(12.1)$ & 3691 (10.9) & $1450(12.2)$ & $1921(28.3)$ & $31343(32.7)$ & 110693 (35.6) & 80635 (38.4) & $211160(56.8)$ \\
\hline \multicolumn{9}{|c|}{ HIV/AIDS at baseline } \\
\hline No/Unknown & 3149 (99.4) & $33150(97.7)$ & $11364(95.6)$ & $6562(96.7)$ & 95664 (99.8) & 308950 (99.4) & $208736(99.4)$ & 370832 (99.8) \\
\hline Yes & $19(0.6)$ & 796 (2.3) & 519 (4.4) & 222 (3.3) & $184(0.2)$ & $1895(0.6)$ & $1220(0.6)$ & $900(0.2)$ \\
\hline
\end{tabular}


Table 3 Characteristics of currently alive HCV testers by HCV status and birth cohort, Canada, BC-HTC, 1990-2013 (Continued)

\begin{tabular}{|c|c|c|c|c|c|c|c|c|}
\hline \multicolumn{9}{|c|}{ HIV/AIDS co-infection (ever) } \\
\hline No/Unknown & $3135(99.0)$ & $32345(95.3)$ & $10810(91.0)$ & $6335(93.4)$ & $95640(99.8)$ & 308728 (99.3) & 208587 (99.4) & $370739(99.7)$ \\
\hline Yes & $33(1.0)$ & $1601(4.7)$ & $1073(9.0)$ & $449(6.6)$ & $208(0.2)$ & $2117(0.7)$ & $1369(0.7)$ & $993(0.3)$ \\
\hline \multicolumn{9}{|l|}{ HBV at baseline } \\
\hline No/Unknown & $3130(98.8)$ & $33529(98.8)$ & $11686(98.3)$ & $6729(99.2)$ & $94660(98.8)$ & 304567 (98.0) & $206304(98.3)$ & $368692(99.2)$ \\
\hline Yes & $38(1.2)$ & $417(1.2)$ & $197(1.7)$ & $55(0.8)$ & $1188(1.2)$ & $6278(2.0)$ & $3652(1.7)$ & $3040(0.8)$ \\
\hline \multicolumn{9}{|l|}{ HBV co-infection (ever) } \\
\hline No/Unknown & $3075(97.1)$ & $32643(96.2)$ & $11330(95.4)$ & $6658(98.1)$ & $94059(98.1)$ & $302130(97.2)$ & 204856 (97.6) & $367414(98.8)$ \\
\hline Yes & $93(2.9)$ & $1303(3.8)$ & $553(4.7)$ & $126(1.9)$ & $1789(1.9)$ & $8715(2.8)$ & $5100(2.4)$ & $4318(1.2)$ \\
\hline \multicolumn{9}{|l|}{ Active TB at baseline } \\
\hline No/Unknown & $3157(99.7)$ & $33890(99.8)$ & $11863(99.8)$ & $6773(99.8)$ & $95584(99.7)$ & 310307 (99.8) & $209650(99.9)$ & $371368(99.9)$ \\
\hline Yes & $11(0.4)$ & $56(0.2)$ & $20(0.2)$ & $11(0.2)$ & $264(0.3)$ & $538(0.2)$ & $306(0.2)$ & $364(0.1)$ \\
\hline \multicolumn{9}{|c|}{ Active TB co-infection (ever) } \\
\hline No/Unknown & $3149(99.4)$ & $33783(99.5)$ & $11808(99.4)$ & $6752(99.5)$ & $95494(99.6)$ & 310145 (99.8) & 209558 (99.8) & $371276(99.9)$ \\
\hline Yes & $19(0.6)$ & $163(0.5)$ & $75(0.6)$ & $32(0.5)$ & $354(0.4)$ & $700(0.2)$ & $398(0.2)$ & $456(0.1)$ \\
\hline \multicolumn{9}{|c|}{ Material deprivation quintile at baseline } \\
\hline Unknown & $106(3.4)$ & $1298(3.8)$ & $618(5.2)$ & $469(6.9)$ & $1199(1.3)$ & $3720(1.2)$ & $2671(1.3)$ & $7183(1.9)$ \\
\hline Q1 (most privileged) & $507(16.0)$ & $4444(13.1)$ & $1362(11.5)$ & $830(12.2)$ & $18845(19.7)$ & $65327(21.0)$ & $48322(23.0)$ & $81513(21.9)$ \\
\hline Q2 & $513(16.2)$ & $5404(15.9)$ & $1636(13.8)$ & $936(13.8)$ & $18165(19.0)$ & $60803(19.6)$ & $39355(18.7)$ & $69264(18.6)$ \\
\hline Q3 & $533(16.8)$ & $6114(18)$ & $1926(16.2)$ & $1065(15.7)$ & $19060(19.9)$ & $61814(19.9)$ & $40022(19.1)$ & $71369(19.2)$ \\
\hline Q4 & $660(20.8)$ & $7365(21.7)$ & $2691(22.7)$ & $1514(22.3)$ & $20032(20.9)$ & $61824(19.9)$ & $40792(19.4)$ & $74468(20.0)$ \\
\hline Q5 (most deprived) & 849 (26.8) & $9321(27.5)$ & $3650(30.7)$ & $1970(29.0)$ & $18547(19.4)$ & $57357(18.5)$ & $38794(18.5)$ & $67935(18.3)$ \\
\hline \multicolumn{9}{|c|}{ Social deprivation quintile at baseline } \\
\hline Unknown & $106(3.4)$ & $1298(3.8)$ & $618(5.2)$ & $469(6.9)$ & $1199(1.3)$ & $3720(1.2)$ & $2671(1.3)$ & $7183(1.9)$ \\
\hline Q1 (most privileged) & $557(17.6)$ & $3477(10.2)$ & $1081(9.1)$ & $741(10.9)$ & $17348(18.1)$ & $57695(18.6)$ & $36550(17.4)$ & $68621(18.5)$ \\
\hline Q2 & $513(16.2)$ & $4335(12.8)$ & $1238(10.4)$ & $794(11.7)$ & $17802(18.6)$ & $56839(18.3)$ & $36109(17.2)$ & $64348(17.3)$ \\
\hline Q3 & $516(16.3)$ & $5802(17.1)$ & $1790(15.1)$ & $989(14.6)$ & $17342(18.1)$ & $56864(18.3)$ & $36204(17.2)$ & $65305(17.6)$ \\
\hline Q4 & $606(19.1)$ & $7152(21.1)$ & $2380(20.0)$ & $1257(18.5)$ & $19201(20.0)$ & $62437(20.1)$ & $42960(20.5)$ & $76953(20.7)$ \\
\hline Q5 (most deprived) & $870(27.5)$ & $11882(35.0)$ & $4776(40.2)$ & $2534(37.4)$ & $22956(24.0)$ & $73290(23.6)$ & $55462(26.4)$ & $89322(24.0)$ \\
\hline \multicolumn{9}{|l|}{ Mental illness at baseline ${ }^{b}$} \\
\hline No/Unknown & $2768(89.8)$ & $28051(85.0)$ & $9468(81.9)$ & $4942(79.5)$ & $75892(90.3)$ & $230429(85.6)$ & $160387(87.7)$ & $262690(89.8)$ \\
\hline Yes & $316(10.2)$ & $4956(15.0)$ & $2087(18.1)$ & $1277(20.5)$ & $8130(9.7)$ & 38636 (14.4) & $22397(12.3)$ & 29891 (10.2) \\
\hline
\end{tabular}


Table 3 Characteristics of currently alive HCV testers by HCV status and birth cohort, Canada, BC-HTC, 1990-2013 (Continued)

\begin{tabular}{|c|c|c|c|c|c|c|c|c|}
\hline \multicolumn{9}{|c|}{ Mental illness 3yr pre- baseline ${ }^{b}$} \\
\hline No/Unknown & $2908(94.3)$ & $30114(91.2)$ & $10261(88.8)$ & $5478(88.1)$ & $80387(95.7)$ & $251001(93.3)$ & $172141(94.2)$ & $277513(94.8)$ \\
\hline Yes & $176(5.7)$ & $2893(8.8)$ & $1294(11.2)$ & $741(11.9)$ & $3635(4.3)$ & $18064(6.7)$ & $10643(5.8)$ & $15068(5.2)$ \\
\hline \multicolumn{9}{|c|}{ Illicit drug use at baseline ${ }^{b}$} \\
\hline No/Unknown & $2763(89.6)$ & $22649(68.6)$ & $6220(53.8)$ & $3256(52.4)$ & $81535(97.0)$ & 249677 (92.8) & $167729(91.8)$ & $271558(92.8)$ \\
\hline Yes & $321(10.4)$ & $10358(31.4)$ & $5335(46.2)$ & $2963(47.6)$ & $2487(3.0)$ & $19388(7.2)$ & $15055(8.2)$ & $21023(7.2)$ \\
\hline \multicolumn{9}{|c|}{ Illicit drug use 3yr pre- baseline ${ }^{b}$} \\
\hline No/Unknown & $2870(93.1)$ & $25193(76.3)$ & $7090(61.4)$ & $3557(57.2)$ & $83133(98.9)$ & $259681(96.5)$ & $174045(95.2)$ & $278466(95.2)$ \\
\hline Yes & $214(6.9)$ & $7814(23.7)$ & 4465 (38.6) & $2662(42.8)$ & $889(1.1)$ & $9384(3.5)$ & $8739(4.8)$ & $14115(4.8)$ \\
\hline \multicolumn{9}{|c|}{ Problematic alcohol use at baseline ${ }^{b}$} \\
\hline No/Unknown & $2724(88.3)$ & $25720(77.9)$ & $8780(76.0)$ & $4933(79.3)$ & $79838(95.0)$ & $248826(92.5)$ & $171200(93.7)$ & $277407(94.8)$ \\
\hline Yes & $360(11.7)$ & $7287(22.1)$ & $2775(24.0)$ & $1286(20.7)$ & $4184(5.0)$ & $20239(7.5)$ & $11584(6.3)$ & $15174(5.2)$ \\
\hline \multicolumn{9}{|c|}{ Problematic alcohol use 3yr pre-baseline ${ }^{b}$} \\
\hline No/Unknown & $2870(93.1)$ & $28620(86.7)$ & $9874(85.5)$ & $5421(87.2)$ & $81897(97.5)$ & $258911(96.2)$ & $177020(96.8)$ & $284664(97.3)$ \\
\hline Yes & 214 (6.9) & 4387 (13.3) & $1681(14.5)$ & 798 (12.8) & 2125 & 10154 (3.8) & 5764 (3.2) & 7917 (2.7) \\
\hline
\end{tabular}

${ }^{a}$ Baseline is defined as date of diagnosis (i.e. first HCV positive test or case report) for HCV positive individuals, and date of last negative test result for HCV negative individuals

${ }^{\mathrm{b}}$ Mental health, drug misuse, alcohol misuse data was available up to 2012 
Table 4 Multivariable multinomial logistic regression models for factors associated with seroconversion and prevalent HCV infection in BC-HTC, Canada 1990-2012 a, b

\begin{tabular}{|c|c|c|}
\hline \multirow[t]{2}{*}{ Variables } & Seroconverters & Prevalent HCV \\
\hline & ORs (95 \% Cl) & ORs (95 \% Cl) \\
\hline \multicolumn{3}{|l|}{ Sex } \\
\hline Female & 1.00 & 1.00 \\
\hline Male & $1.25(1.19-1.31)$ & $2.06(2.02-2.10)$ \\
\hline \multicolumn{3}{|l|}{ Birth year } \\
\hline$<1945$ & 1.00 & 1.00 \\
\hline 1945-1954 & $3.38(2.78-4.11)$ & $3.79(3.67-3.92)$ \\
\hline 1955-1964 & $6.22(5.19-7.44)$ & $3.23(3.13-3.34)$ \\
\hline 1965-1974 & $9.86(8.26-11.78)$ & $1.64(1.58-1.70)$ \\
\hline 1975-1984 & $11.76(9.83-14.07)$ & $0.70(0.67-0.73)$ \\
\hline$\geq 1985$ & $6.53(5.34-7.99)$ & $0.48(0.44-0.51)$ \\
\hline \multicolumn{3}{|l|}{ HIV infection at baseline ${ }^{c}$} \\
\hline No/Unknown & 1.00 & 1.00 \\
\hline Yes & $7.92(7.05-8.89)$ & $4.80(4.47-5.15)$ \\
\hline \multicolumn{3}{|l|}{ HBV at baseline ${ }^{c}$} \\
\hline No/Unknown & 1.00 & 1.00 \\
\hline Yes & $2.33(2.00-2.71)$ & $0.69(0.63-0.75)$ \\
\hline \multicolumn{3}{|l|}{ Active TB at baseline $^{c}$} \\
\hline No/Unknown & 1.00 & 1.00 \\
\hline Yes & $1.00(0.62-1.62)$ & $1.01(0.81-1.25)$ \\
\hline \multicolumn{3}{|c|}{$\begin{array}{l}\text { Material deprivation quintile at } \\
\text { baseline }^{c}\end{array}$} \\
\hline Unknown & $3.51(2.96-4.17)$ & $5.38(5.07-5.70)$ \\
\hline Q1 (most privileged) & 1.00 & 1.00 \\
\hline Q2 & $1.20(1.06-1.31)$ & $1.22(1.18-1.27)$ \\
\hline Q3 & $1.24(1.13-1.36)$ & $1.36(1.32-1.41)$ \\
\hline Q4 & $1.62(1.48-1.76)$ & $1.60(1.55-1.65)$ \\
\hline Q5 (most deprived) & $1.99(1.83-2.15)$ & $1.98(1.92-2.04)$ \\
\hline \multicolumn{3}{|c|}{ Mental illness at baseline ${ }^{c}$} \\
\hline No/Unknown & 1.00 & 1.00 \\
\hline Yes & $1.18(1.12-1.25)$ & $0.69(0.67-0.72)$ \\
\hline \multicolumn{3}{|c|}{ Illicit drugs use at baseline } \\
\hline No/Unknown & 1.00 & 1.00 \\
\hline Yes & $21.00(19.80-22.38)$ & $5.03(4.91-5.16)$ \\
\hline \multicolumn{3}{|c|}{$\begin{array}{l}\text { Problematic alcohol use at } \\
\text { baseline }\end{array}$} \\
\hline No/Unknown & 1.00 & 1.00 \\
\hline Yes & $2.11(2.00-2.23)$ & $1.67(1.63-1.72)$ \\
\hline
\end{tabular}

${ }^{a}$ Adjusted for health authority and year of diagnosis; ${ }^{\text {b Excluding unknown }}$ gender and health authority. ${ }^{\mathrm{C} B a s e l i n e}$ is defined as time of diagnosis for HCV positive, and date of last negative for HCV negative

studies on feasibility and cost effectiveness of various strategies are needed to identify undiagnosed infections in $\mathrm{BC}$.
Risk factors for both new and prevalent HCV infections were similar with some notable exceptions, including age, birth cohort, drug use, HBV co-infection and mental health diagnoses. HBV co-infection and mental health problems were significantly associated with increased odds of new infection but decreased odds of prevalent $\mathrm{HCV}$ infection, while ORs for illicit drug use compared to no drug use among new infections $(\mathrm{AOR}=$ 21) were four times than those for prevalent $\mathrm{HCV}$ infection (AOR $=5.0)$. ORs for prevalent HCV infection were highest for birth cohort 1945-64 and declined thereafter, while among new infections, ORs were highest for those born later, consistent with an earlier analysis demonstrating a higher HCV incidence rate in younger birth cohorts [25]. Demographic characteristics and risk factors for prevalent $\mathrm{HCV}$ were also consistent with recent data from the United States and Canada [8, 20]. Likewise, in a recent electronic medical record based study from the US, being a baby boomer, male, people who injects drugs (PWID), HIV co-infected and having low income were associated with HCV positivity [26]. Risk factors among new infections were also similar to those identified with $\mathrm{HCV}$ infection in high risk populations, mainly PWID. Among seroconverters especially 24months seroconverters, those born 1965-84, HIV/HBV co-infection, socioeconomic deprivation, mental illness, illicit drug use and problematic alcohol use were more common than among prevalent HCV infections (Table 1/ Fig. 1). These findings highlight the presence of syndemics of blood-borne infections (BBI), mental illness, addiction, and socioeconomic marginalization in this population as has been reported by others [27-29]. Addressing these syndemics requires comprehensive services including integrated testing, prevention and treatment for STI/BBIs, as well as mental health and addiction services to address the needs of this population group.

Findings from this paper should be interpreted in the context of some methodological issues. The validity of our estimates depends on the successful linkage rate. Linkage rates were very high for $\mathrm{HCV}$ (>85\%), especially in recent years [14]. Linkage rates for those HIV co-infected were much higher than the overall HIV linkage rate, especially before 2005. Thus, the HIV co-infection rate may have been underestimated. However, as reported earlier, we used multiple sources of HIV status identification to reduce underestimation. In this study, we did not have access to immigration and Aboriginal status data and hence we were not able to characterize the disease burden among immigrants and Aboriginal populations. Other data suggest that Aboriginal people are five times more likely to be infected with $\mathrm{HCV}$ [30]. Immigrants from endemic countries are also more likely to have higher $\mathrm{HCV}$ infection rates. 
To date, laboratory testing for HCV has followed risk based guidelines and hence a higher positivity rate among HCV testers in this cohort is expected compared to the general population. However, general population surveys such as the Canadian Health Measures Survey may not capture high risk populations with higher $\mathrm{HCV}$ prevalence thus and may underestimate total population of infected individuals $[8,20]$. Categorizing prevalent infections based on being positive at the first test may be an over-estimate as some of these individuals are likely to be recent seroconverters as shown in our recent molecular analysis [31]. The prevalent HCV case detection could be affected by presence of late stage symptomatic disease or survival. Survival may also affect the difference in risk factor profile between prevalent and new cases. In the BC-HTC, data is available on all cohort members many years before cohort initiation to assess risk factors as presented in Table 1. Thus, for all cohort members regardless of first test or diagnosis date, data on risk factors started at the same time, providing ample time to assess risk factors from the available data. Furthermore, after accounting for mortality in the cohort, there was no difference in identified risk factor patterns in models including both currently alive and dead and only those currently who are currently alive. Thus, survival bias is unlikely to explain difference in risk factor profile between prevalent and new infections. However, survival bias is not expected to be completely eliminated especially in early nineties, when people could have died before $\mathrm{HCV}$ diagnosis or getting diagnosed because of symptoms related to late stage disease. In another analysis, we found that late $\mathrm{HCV}$ diagnosis in relation to advance stage liver disease (hepatocellular carcinoma and decompensated cirrhosis) was common in early nineties and have declined substantially over time [32].

In the current paper, data on RNA testing and active infection was not presented, which is important to assess people living with active infection and need treatment to prevent progressive liver disease. The $\mathrm{BC}$-HTC provides a platform to assess program progress through cascade of care monitoring, long term outcomes related to $\mathrm{HCV}$ and impact of treatment on long term outcomes. These data are in the process of being analyzed and will be presented in future reports.

\section{Conclusions}

The HCV positivity rate was highest in the 1950-54 and 1955-59 birth cohorts and overall among those born between 1945-1964, which declined over time. Furthermore, the year over year decline in the positivity rate suggests that most of the $\mathrm{HCV}$ infections in these cohorts have already been identified. However, current risk based testing may not identify individuals who are unable to recall or are unwilling to disclose remote risk behaviors. Further studies are needed to estimate the number of undiagnosed HCV infection and assess optimal strategies to identify the remaining undiagnosed infections. Newly acquired infections are occurring mainly in younger birth cohorts and these groups are more likely to be co-infected with HIV and/or HBV, socioeconomically marginalized, and living with serious mental illnesses and addictions. Comprehensive syndemic approaches that take into account co-infections, mental health, additions and socioeconomic vulnerabilities are urgently required to identify, treat, and support people with $\mathrm{HCV}$ infection.

\section{Additional file}

\begin{abstract}
Additional file 1: Table S1. Definitions for comorbid conditions. Table S2. Distribution HCV seroconverters by seroconversion interval in BC HTC, Canada 1990-2013. Table S3. Characteristics of currently alive recently diagnosed (2010-2013) HCV positive and negative individuals, BC-HTC, 2010 - 2013. Table S4. Percentage positive for hepatitis $C$ by diagnosis year and birth cohort in British Columbia, 1992-2013. Figure S1. Hepatitis C percentage positive by year of diagnosis and birth cohort, BC-HTC, British Columbia, Canada, 2000-2013. Table S5. Multivariable multinomial logistic regression model for factors associated with seroconversion and chronic HCV infection including age as covariate in BC HTC, Canada 1990-2012 a,b. Table S6. Multivariable multinomial logistic regression model for factors associated with seroconversion and chronic HCV infection including indicators for recent risk activities in BC HTC, Canada 1990-2012 a,b. (DOCX 68 kb)
\end{abstract}

\section{Abbreviations \\ BC-HTC, The BC Hepatitis Testers Cohort; HCV, hepatitis C virus; BC, British Columbia; HIV, Human immunodeficiency virus; HBV, hepatitis B virus; TB, Tuberculosis; STIs, Sexually transmitted infections; BCCDC, British Columbia Center for Disease Control; BBI, Bloodborne infection; PWID, People who injection drugs}

\section{Acknowledgements}

We acknowledge the assistance of BCCDC, PHSA Performance measurement and reporting, Information Analysts, Ministry of Health Data Access, Research and Stewardship, \& MSP, DAD and Medical Beneficiary and Pharmaceutical Services programme areas, BC Ministry of Health, and BC Cancer Agency and their staff involved in data access and procurement, and data management.

\section{Funding}

This work was supported by BC Centre for Disease Control and Agencies contributing data to the study and the Canadian Institutes of Health Research [Grant \# 201503NHC-348216-NHC-ADWY -62134 \& 201410PHE-337680-PHECAAA-179547].

\section{Availability of data and materials}

The study is based on data linkage that is available through respective data stewards. Information on data access is available at BC Centre for Disease Control at following link: http://www.bccdc.ca/about/accountability/dataaccess-requests/public-health-data.

\section{Authors' contributions}

NZJ conceived of the study, participated in its design, guided the statistical analysis, wrote first draft, and revised the manuscript. AY participated in the design of the study and performed the statistical analysis. MKu and MA participated in the design of the study and helped to draft the manuscript. DC, JW and MT helped to draft the manuscript. MKr participated in the design of the study, interpretation of findings and helped to draft the manuscript. All authors read and approved the final manuscript. 


\section{Competing interests}

MK has received grant funding via his institution from Roche Molecular Systems, Boehringer Ingelheim, Merck, Siemens Healthcare Diagnostics and Hologic Inc.

\section{Consent for publication}

Not Applicable

\section{Ethics and consent to participate}

Data linkage to establish the BC-HTC was performed under the BCCDC's public health mandate. The Behavioral Research Ethics Board at the University of British Columbia reviewed and approved the study (H14-01649). Patient consent was not required as study used de-identified linked administrative healthcare datasets.

\section{Author details}

'Clinical Prevention Services, British Columbia Centre for Disease Control, Vancouver, BC, Canada. ${ }^{2}$ School of Population and Public Health, University of British Columbia, Vancouver, BC, Canada. ${ }^{3}$ Department of Pathology and Laboratory Medicine, University of British Columbia, Vancouver, BC, Canada.

Received: 12 January 2016 Accepted: 13 June 2016

Published online: 19 July 2016

\section{References}

1. Mohd Hanafiah K, Groeger J, Flaxman AD, Wiersma ST. Global epidemiology of hepatitis $C$ virus infection: new estimates of age-specific antibody to HCV seroprevalence. Hepatology. 2013;57(4):1333-42.

2. Trubnikov M, Yan P, Archibald C. Estimated prevalence of Hepatitis $C$ virus infection in Canada, 2011. CCDR. 2014;40(19):429-42.

3. Communicable Disease Prevention and Control Services. British Columbia Annual Summary of Reportable Diseases 2013. Vancouver: BC Centre for Disease Control; 2014.

4. Yu A, Spinelli JJ, Cook DA, Buxton JA, Krajden M. Mortality among British Columbians testing for hepatitis C antibody. BMC Public Health. 2013;13:291.

5. Simmons B, Saleem J, Heath K, Cooke GS, Hill A. Long-Term Treatment Outcomes of Patients Infected With Hepatitis C Virus: A Systematic Review and Meta-analysis of the Survival Benefit of Achieving a Sustained Virological Response. Clinical Infect Dis. 2015;61(5):730-40.

6. Kohli A, Shaffer A, Sherman A, Kottilil S. Treatment of hepatitis C: a systematic review. JAMA. 2014:312(6):631-40.

7. Canadian Agency for Drugs and Technologies in Health. Holkira (Ombitasvir/Paritaprevir/Ritonavir with Dasabuvir) and Harvoni (Ledipasvir/ Sofosbuvir) for Chronic Hepatitis C: A Review of the Clinical Evidence. Canadian Agency for Drugs and Technologies in Health. Ottawa (ON); 2015.

8. Rotermann M, Langlois K, Andonov A, Trubnikov M. Seroprevalence of hepatitis B and C virus infections: Results from the 2007 to 2009 and 2009 to 2011 Canadian Health Measures Survey. Health Rep. 2013;24(11):3-13.

9. BC Cancer Agency [creator]. BC Cancer Agency Registry Data (2013). Data Extract. Vancouver, BC: Cancer Agency [publisher]; 2014.

10. British Columbia Ministry of Health [creator]. PharmaNet. Victoria, BC: British Columbia Ministry of Health [publisher]; Data Extract. MOH (2013); 2014. http://www.health.gov.bc.ca/data/.

11. British Columbia Ministry of Health [creator]. Medical Services Plan (MSP) Payment Information File. Victoria, BC: British Columbia Ministry of Health [publisher]; Data Extract. MOH (2013). 2014. http://www.health.gov.bc.ca/data/.

12. British Columbia Ministry of Health [creator]. Discharge Abstract Database (Hospital Separations). Victoria, BC: British Columbia Ministry of Health [publisher]. Data Extract. MOH (2013). 2014. http://www.health.gov.bc.ca/data/.

13. British Columbia Vital Statistics Agency [creator]. Vital Statistics Deaths. Victoria, BC: British Columbia Vital Statistics Agency [publisher]. Data Extract. British Columbia Vital Statistics Agency (2014). 2014.

14. Janjua NZ, Kuo M, Chong M, Yu A, Alvarez M, Cook D, Armour R, Aiken C, Li K, Mussavi Rizi SA et al. Assessing Hepatitis C Burden and Treatment Effectiveness through the British Columbia Hepatitis Testers Cohort (BC-HTC): Design and Characteristics of Linked and Unlinked Participants. PLoS One. 2016;11(3): e0150176.

15. BC Centre for Disease Control. Management of Specific Diseases: Hepatitis C. In: Communicable Disease Control Manual. Vancouver: BC Centre for Disease Control; 2013.
16. Pampalon R, Hamel D, Gamache P, Philibert MD, Raymond G, Simpson A. An area-based material and social deprivation index for public health in Quebec and Canada. Can J Public Health. 2012;103(8 Suppl 2):S17-22.

17. Environics Analytics. 2011 CensusPlus. Edited by Environics Analytics. Toronto, ON 2013.

18. McGuinness L. BCCDC Enhanced Hepatitis Strain Surveillance System (EHSSS) Site Report 2000-2011. Vancouver: BC Centre for Disease Control; 2014.

19. Armstrong GL, Wasley A, Simard EP, McQuillan GM, Kuhnert WL, Alter MJ. The prevalence of hepatitis C virus infection in the United States, 1999 through 2002. Ann Intern Med. 2006;144(10):705-14.

20. Denniston MM, Jiles RB, Drobeniuc J, Klevens RM, Ward JW, McQuillan GM, Holmberg SD. Chronic hepatitis C virus infection in the United States, National Health and Nutrition Examination Survey 2003 to 2010. Ann Intern Med. 2014; 160(5):293-300.

21. Galbraith JW, Franco RA, Donnelly JP, Rodgers JB, Morgan JM, Viles AF, Overton ET, Saag MS, Wang HE. Unrecognized chronic hepatitis C virus infection among baby boomers in the emergency department. Hepatology. 2015;61(3):776-82.

22. Smith BD, Morgan RL, Beckett GA, Falck-Ytter Y, Holtzman D, Ward JW. Hepatitis C virus testing of persons born during 1945-1965: recommendations from the Centers for Disease Control and Prevention. Ann Intern Med. 2012;157(11):817-22.

23. Schanzer DL, Paquette D, Lix LM. Historical trends and projected hospital admissions for chronic hepatitis C infection in Canada: a birth cohort analysis. CMAJ Open. 2014;2(3):E139-44.

24. Health Canada. Guidelines and recommendations on the prevention and control of hepatitis C. Can Commun Dis Rep 1995, 21 Suppl 2:1-18, 11-21.

25. Kuo M, Janjua NZ, Burchell AN, Buxton JA, Krajden M, Gilbert M. Decreasing Hepatitis C Incidence Among a Population With Repeated Tests: British Columbia, Canada, 1993-2011. Am J Public Health. 2015;105(8):1604-10.

26. Smith BD, Yartel AK, Krauskopf K, Massoud OI, Brown KA, Fallon MB, Rein DB. Hepatitis $C$ virus antibody positivity and predictors among previously undiagnosed adult primary care outpatients: cross-sectional analysis of a multisite retrospective cohort study. Clin Infect Dis. 2015;60(8):1145-52.

27. Akselrod H, Grau LE, Barbour R, Heimer R. Seroprevalence of HIV, hepatitis B virus, and HCV among injection drug users in Connecticut: understanding infection and coinfection risks in a nonurban population. Am J Public Health. 2014;104(9):1713-21.

28. Klein MB, Rollet KC, Saeed S, Cox J, Potter M, Cohen J, Conway B, Cooper C, Cote P, Gill J et al. HIV and hepatitis C virus coinfection in Canada: challenges and opportunities for reducing preventable morbidity and mortality. HIV Med. 2013;14(1):10-20.

29. Morano JP, Gibson BA, Altice FL. The burgeoning HIV/HCV syndemic in the urban Northeast: HCV, HIV, and HIV/HCV coinfection in an urban setting. PLoS One. 2013;8(5):e64321.

30. Public Health Agency of Canada. Epidemiology of acute hepatitis C infection in Canada: results from the enhanced hepatitis strain surveillance system (EHSSS). 2009.

31. Olmstead AD, Joy JB, Montoya V, Luo I, Poon AF, Jacka B, Lamoury F, Applegate T, Montaner J, Khudyakov $Y$ et al. A molecular phylogeneticsbased approach for identifying recent hepatitis $\mathrm{C}$ virus transmission events. Infect Genet Evol. 2015;33:101-9.

32. Janjua NZ, Yu A, Kuo M, Alavi M, Woods R, Alvarez M, Dore G, Tyndall M, Krajden M. Timing of Hepatitis B and C Diagnosis Relative to Hepatocellular Carcinoma Diagnosis in the BC Hepatitis Testers Cohort (BC-HTC). Hepatology. 2015;62(S1):480A.

\section{Submit your next manuscript to BioMed Central} and we will help you at every step:

- We accept pre-submission inquiries

- Our selector tool helps you to find the most relevant journal

- We provide round the clock customer support

- Convenient online submission

- Thorough peer review

- Inclusion in PubMed and all major indexing services

- Maximum visibility for your research

Submit your manuscript at www.biomedcentral.com/submit 